\title{
Bayesian Dynamic Linear Models for Estimation of Phenological Events from Remote Sensing Data
}

\begin{abstract}
Estimating the timing of the occurrence of events that characterize growth cycles in vegetation from time series of remote sensing data is desirable for a wide area of applications. For example, the timings of plant life cycle events are very sensitive to weather conditions, and are often used to assess the impacts of changes in weather and climate. Likewise, understanding crop phenology can have a large impact on agricultural strategies. To study phenology using remote sensing data, the timings of annual phenological events must be estimated from noisy time series that may have many missing values. Many current state-of-the-art methods consist of smoothing time series and estimating events as features of smoothed curves. A shortcoming of many of these methods is that they do not easily handle missing values and require imputation as a pre-processing step. In addition, while some currently used methods may be extendable to allow for temporal uncertainty quantification, uncertainty intervals are not usually provided with phenological event estimates. We propose methodology utilizing Bayesian dynamic linear models to estimate the timing of key phenological events from remote sensing data with uncertainty intervals. We illustrate the methodology on weekly vegetation index data from 2003 to 2007 over a region of southern India, focusing on estimating the timing of start of season (SOS) and peak of greenness (POG). Additionally, we present methods utilizing the Bayesian formulation and MCMC simulation of the model to estimate the probability that more than one growing season occurred in a given year.
\end{abstract}

KEYWORDS: land surface phenology; time series; uncertainty quantification 


\section{Introduction}

Plant phenology is the study of variation in recurring biological cycles in vegetation defined by the occurrence of key life cycle events, such as bud-burst, first flowering, and leaf fall (http: //www. usanpn. org/). The timings of life cycle events are sensitive to changes in weather and climate, and can therefore be used as key indicators of the effects of climate change. As climate is a regional or global process, this requires historical characterization of vegetation life cycles on a large scale. Additionally, crop phenology can be used to monitor crop yield, growing conditions, etc. and could therefore play a large role in optimizing agricultural practices (Duncan et al. 2015). However, events such as bud-burst or first flowering can only be observed using in situ (ground) observations, and consequently, studies of long-term phenology from ground observations cannot feasibly be conducted on a global scale. Fortunately, many vegetation types have life cycles marked by recurring changes that are reflected in properties of the Earth's surface (for example, changes in photosynthetic activity). These can often be identified as changes in the reflectance of the land surface and can therefore be measured using remote sensing data acquired by satellites (Hanes et al. 2014). The study of the timing of recurring seasonal changes in surface vegetation, as measured by remote sensing is called land surface phenology (LSP).

Imaging instruments housed on satellites are used to repeatedly collect data on spectral reflectance of the Earth's surface over time, resulting in time series of satellite sensor imagery. The most common sources of these data are the Advanced Very High Resolution Radiometer (AVHRR), SPOT-VEGETATION (SPT-VGT), Moderate-Resolution Imaging Spectroradiometer (MODIS) and MEdium Resolution Imaging Spectrometer (MERIS) (De Beurs and Henebry 2010; Duncan et al. 2015). A measure of vegetation is usually characterized by vegetation indices (we will refer to these throughout the manuscript as VIs), which are scientifically derived as functions of observed reflectance from various spectral bands (Duncan et al.2015). Commonly used VIs include the red edge position, the Normalized Difference Vegetation Index (NDVI), and the MERIS Terrestrial Chlorophyll Index (MTCI). However, a major challenge in utilizing satellite sensor data is that reflectance from the Earth's surface is often not perfectly measured by the imaging instru- 
ment due to factors such as cloud cover, atmospheric interference and soil background. Because of this, satellite sensor data and derived VIs are often noisy and have many missing values.

Additionally, remote sensing data is constrained to the spatial resolution of the satellite sensors and therefore does not directly provide information about ground based, species level phenology such as first flowering or bud-burst. Consequently, land surface phenology requires that key LSP metrics (or phenological events) characterizing vegetation life cycles be defined and identified from time series of VIs. Examples of phenological events of interest are: timing of onset of greenness or start of season, timing of peak greenness or maximal growth, timing of end of senescence or end of season, and duration of the growing season (De Beurs and Henebry 2010).

Therefore, special attention has been given to developing methods for scientifically accurate identification of phenological events from noisy time series of satellite derived VIs. Such methods include setting thresholds on VIs, derivative based largest increase/decrease in VI, moving averages, and smoothing based model fitting methods (De Beurs and Henebry 2010). Threshold and derivative based methods on raw VIs cannot easily handle multiple growth cycles, nor do they have probabilistic error structures which makes uncertainty assessment infeasible (De Beurs and Henebry 2010; Zhang et al. 2003).

In this work, we approach the problem by identifying a model for the time series of VIs representing the underlying seasonal growth cycles. An important feature of this approach is that it yields a smooth curve which can be used to identify phenological events based on rules defining the events as features of the smoothed time series (e.g. local minima and maxima (Jeganathan et al. 2010b; Dash et al. 2010), thresholds (Prasad and Hegde 1986), and derivative based methods (De Beurs and Henebry 2010), among others). There are currently no universally agreed upon rules to identify phenological events from smoothed VI time series.

In addition to providing a good representation of the growth cycles, smoothing methods should 1) be sufficiently flexible to allow for inter-annual variability in seasons, and 2) allow for multiple growth cycles due to double or triple cropping agriculture, multiple rainy seasons, etc. (De Beurs and Henebry 2010; Zhang et al. 2003). Various methods have been used by applied scientists to smooth remote sensing data for phenological event estimation. For example, the U.S. Geological 
Survey (USGS) methodology for studying land surface phenology utilizes a least squares moving window approach on NDVI data which requires fitting a regression line within the window at each time point. These regression lines are then averaged at each time point to construct a smooth curve from which to identify various phenological events (http://phenology.cr.usgs.gov/). Studies utilizing discrete Fourier transforms that retain only a sufficient number of harmonics to smooth the time series and to allow for multiple growing seasons per year have also become increasingly popular (Jeganathan et al. 2010a b; Dash et al. 2010; Atkinson et al. 2012; Geerken et al. 2005; Geerken 2009). In this approach, inter-annual flexibility is only possible by applying the Fourier transform separately to yearly intervals of data. Other common smoothing methods in the applied literature utilize Savitzy-Golay filters, piecewise fitting using local functions, and wavelet transforms, among others (Jönsson and Eklundh 2004; Zhang et al. 2003; O'Connor et al. 2012; Kandasamy and Fernandes 2015; Atkinson et al. 2012; Tan et al. 2011; Sakamoto et al. 2005; Duncan et al. 2015). It is important to note that none of these approaches provide a natural environment for quantifying the uncertainty associated with event identification. This can be problematic, as the timings of phenological events estimated from remote sensing data are then incorporated like data observed without error in environmental and agricultural studies.

An additional, major challenge in phenological event estimation arises when the VI time series exhibit more than one growth cycle per year. One of the driving motivations of land surface phenology is to characterize phenology in areas where ground observations are not available, and as such the "true" number of growing seasons that should be exhibited is also not available. At the spatial resolution of the satellite, land cover information is generally too coarse to provide a reasonable indication of the expected number of growing seasons (e.g. natural vegetation versus predominantly agriculture). Additionally, deforestation and changes in agricultural practices may change the number of growing seasons present in a region from one year to the next. Most smoothing based methodologies currently employed are flexible enough to model more than one peak, but determination of when a minor peak is a true secondary (or tertiary) growth cycle (as opposed to noise, a rainy season, etc.) is difficult. Identification of secondary growing seasons generally requires the specification of scientifically driven, but relatively ad hoc, conditions that must be sat- 
isfied, such as a minimal change in minimum and maximum growth in a season (see for example Dash et al. (2010) and Vrieling et al. (2011; 2013)).

In this manuscript, we present a new method for smoothing and phenological event estimation utilizing a Bayesian dynamic linear modeling framework, aimed at addressing some of the current challenges in characterizing phenology from remote sensing data. We provide a formal approach to model seasonal growth in vegetation by incorporating dynamic Fourier harmonic terms, allowing seasonality to vary smoothly across years, unlike the simpler harmonic models fitted separately by year in Jeganathan et al. (2010a; 2010b), Atkinson et al. (2012) and Dash et al. (2010). Utilizing a single model for the entire time domain, allowing for seasonality to vary across years by incorporating temporal dependence, and the ability to apply the method universally across regions with vastly varying phenological structure are some of the major advantages offered by our proposed approach. Additionally, the estimation procedures of dynamic linear models can naturally handle missing values, eliminating the pre-processing data imputation step required by many of the current methodologies. Lastly, we introduce a novel approach to determine the number of growing seasons in a given year and assess temporal uncertainty in phenological events by using a fully Bayesian formulation and MCMC simulation of the model. In Section 5 we discuss potential extensions of our method to simultaneously fitting a space-time dynamic model across all locations, building on substantial literature in this field, such as Cressie and Wikle (2011), Banerjee et al. (2014), Gamerman (2010), and Gelfand and Banerjee (2017), including discussing some challenges in our application area such as potentially abrupt land-use changes and non-stationarity both in space and in time. Our primary goal in this manuscript is estimating and quantifying temporal uncertainty in location-specific timings of phenological events, with emphasis on events occurring in time. For this reason we have concentrated on the temporal structure, recognizing that substantial open questions remain in fully taking spatial structure into account.

\section{Data}

The VI used in this work is the MERIS Terrestrial Chlorophyll Index (MTCI), which is derived from spectral reflectance captured by the MERIS satellite. The index is considered to be directly 
related to a measure of canopy chlorophyll content and was designed to have limited sensitivity to atmospheric effects and soil background interference (Dash and Curran 2004, 2007). Dash, Jeganathan and Atkinson have undertaken several studies utilizing MTCI to study phenology over India, which also motivates this work (Dash et al. 2010; Jeganathan et al. 2010a b; Atkinson et al. 2012).

We considered 8-day temporal composites of the MTCI level-3 product over the southern tip of India. The data span the period 2003-2007 and are available on a regular grid at $4.6 \mathrm{~km}$ spatial resolution. We consider a subregion of the data corresponding to a 50x50 lattice containing 2163 "non-sea" grid cells. The temporal resolution of the data gives 46 layers of MTCI each year, resulting in time series of length $T=230$ at each spatial grid cell. The data can be downloaded from the NERC Earth Observation Data Centre website (www.neodc.rl.ac.uk/). Each of the 2163 non-sea time series contains missing values, ranging from $\approx 1.7 \%$ to $\approx 17.9 \%$ missing, with some locations exhibiting periods of as long as 7 consecutive missing composites.

In addition to the MTCI data, the GLC2000 land cover product is used to identify a main type of vegetation for each spatial location. The GLC2000 land cover product was derived using data from the SPOT-4 Vegetation Sensor (Agrawal et al. 2003), and can be downloaded from the European Commission's Joint Research Centre (JRC) website (http: / / forobs • jrc .ec. europa.eu/products/glc2000/products.php). The region considered covers a diverse range of vegetation types. The land cover of the eastern half is primarily agricultural, and the western half is mainly natural vegetation. Figure 1 presents the data from two predominantly natural vegetation locations: (a) corresponds to a primarily Tropical Evergreen land cover location with fairly regular seasonality, while (b) is primarily an area of Tropical Semievergreen and exhibits inter-annual variability in seasonal structure. These two locations will be used to illustrate the proposed methodology throughout the paper. 


\section{Methodology}

\subsection{Dynamic Linear Models}

Dynamic linear models (DLMs) (see, for example, West and Harrison (1999)) are a large, flexible class of non-stationary time series models. They are flexible in that they allow an interpretable decomposition of the series into terms of trend and seasonality, handle missing values, and permit forecasting as well as retrospective analysis of the temporal dynamics. An important advantage of the DLM approach for our application is that it provides a natural environment for uncertainty quantification of identified phenological events.

A univariate DLM assumes that a time series, $\boldsymbol{Y}_{1: T}=\left(Y_{t}: t=1,2, \ldots, T\right)$, of length $T$, is an observed realization of an unobserved latent process on a $p$-dimensional state vector, $\boldsymbol{\theta}_{t}$, subject to Gaussian random noise (for notational clarity, let $\boldsymbol{\theta}_{1: T}=\left(\boldsymbol{\theta}_{t}: t=1,2, \ldots, T\right)$ ). This model has three primary components, namely the:

$$
\begin{array}{crl}
\text { Observation equation : } Y_{t}=\boldsymbol{F}_{t} \boldsymbol{\theta}_{t}+v_{t} & v_{t} \sim N\left(0, V_{t}\right) \\
\qquad \text { State equation : } \boldsymbol{\theta}_{t}=\boldsymbol{G}_{t} \boldsymbol{\theta}_{t-1}+\boldsymbol{w}_{t} & \boldsymbol{w}_{t} \sim N_{p}\left(\mathbf{0}, \boldsymbol{W}_{t}\right) \\
\text { Prior on initial state : } \boldsymbol{\theta}_{0} \sim N_{p}\left(\boldsymbol{m}_{0}, \boldsymbol{C}_{0}\right) . &
\end{array}
$$

Equation (1a) defines how the observed data relate to the unknown (latent) state vector, with $\boldsymbol{F}_{t}$ a $1 \times p$ matrix of (typically known) constants, and $\left(v_{t}\right)_{t \geq 1}$ a sequence of independent observation errors. Equation (1b) defines how the state evolves from time $t-1$ to $t$, and is called the state, system, or evolution equation (Petris et al. 2009). $\boldsymbol{G}_{t}$ is a $p \times p$ matrix of (typically known) constants, and $\left(\boldsymbol{w}_{t}\right)_{t \geq 1}$ is an independent sequence of random vectors, also assumed independent of $\left(v_{t}\right)_{t \geq 1}$. Lastly, $1 \mathrm{c}$ specifies the prior distribution on the state parameter vector at time zero, assumed independent of $\left(v_{t}\right)_{t \geq 1}$ and $\left(\boldsymbol{w}_{t}\right)_{t \geq 1}$. We refer to $\left(V_{t}\right)_{t \geq 1}$ and $\left(\boldsymbol{W}_{t}\right)_{t \geq 1}$ as the observation and evolution variances, respectively, both of which typically are unknown, so a fully specified Bayesian DLM also requires specification of prior distributions on these unknown observation and evolution variances. DLMs are more flexible than classical regression models, but are still a naturally intuitive extension. For example, setting $\boldsymbol{G}_{t}=\boldsymbol{I}_{p}$ and $\boldsymbol{W}_{t}=\mathbf{0}_{p}$, reduces $1 \mathrm{a}, 1 \mathrm{~b}$ ) to the 
linear regression model, $Y_{t}=\boldsymbol{F}_{t} \boldsymbol{\theta}_{0}+v_{t}$.

In the current application, we assume that the latent state process, $\boldsymbol{F}_{t} \boldsymbol{\theta}_{t}, t=1, \ldots, T$, represents the true vegetation growth. Therefore, inference about linear combinations of the state vector $\boldsymbol{\theta}_{0: T}$ is of primary interest. The DLM framework permits three types of posterior inferences about the state vector at time $t^{\prime}$ from $\pi\left(\boldsymbol{\theta}_{t^{\prime}} \mid \boldsymbol{y}_{1: t}\right)$ : filtering (when $t^{\prime}=t$ ), forecasting (when $t^{\prime}>t$ ), and smoothing (when $t^{\prime}<t=T$ ). Of the three, smoothing is a retrospective technique used to study the historical evolution of the system $\boldsymbol{\theta}_{t^{\prime}}$ using all the data available up to and including time $T$, which coincides with our scientific objective of characterizing phenology from historical data. Given $\left(V_{t}\right)_{t \geq 1}$ and $\left(\boldsymbol{W}_{t}\right)_{t \geq 1}$, the smoothing distributions can be determined sequentially using the well-known Kalman recursions (Kalman|1960).

The Bayesian framework for DLMs provides several key advantages over many of the currently used methodologies for characterizing phenology from remote sensing data. One major strength is the ability to easily handle missing values (see, for example, (Petris et al. 2009)). The analyses based on wavelets and Fourier transforms impute missing values as a data pre-processing step, particularly when data are missing at more than one consecutive time point. By contrast, in the Kalman Filter, if $y_{t}$ is missing, it is assumed to carry no information and the filtered distribution, $\pi\left(\theta_{t} \mid y_{1: t}\right)$, is set to the one-step ahead predictive distribution, $\pi\left(\theta_{t} \mid y_{1:(t-1)}\right)$. The dependence structure of the DLM allows past and future data to inform the smoothing distribution of the state process, $\boldsymbol{\theta}_{t}$, when data are missing at time $t$, thus eliminating the need for the often arbitrary pre-processing imputation step.

\subsubsection{Bayesian reduced Fourier-form Dynamic Linear Model}

We choose Bayesian reduced Fourier-form seasonal DLMs to model the time series of satellitebased VIs, thereby using models that are direct extensions of the currently applied Fourier transform methods (Dash et al. 2010; Jeganathan et al. 2010a|b). These Fourier-form DLMs define states as the $s / 2(s$ even) or $(s-1) / 2(s$ odd) Fourier harmonics, with $s$ being the period of the time series, but only retain a small subset, $q$, of these Fourier harmonics in their reduced form (Petris et al. 2009; West and Harrison|1999). They are well suited for our study since they provide smooth representations of time-evolving seasonal patterns in phenology in our region where no 
more than a triple cropping season is expected.

We let $\boldsymbol{Y}_{1: T}=\left(Y_{t}^{*}-\bar{Y}^{*}: t=1,2, \ldots, T\right)$ (where $Y_{t}^{*}$ represents the raw data and $\bar{Y}^{*}=$ $\frac{1}{\left|\boldsymbol{T}^{*}\right|} \sum_{t \in \boldsymbol{T}^{*}} Y_{t}^{*}$ for $\boldsymbol{T}^{*}$, the set of times such that $Y_{t}^{*}$ is non-missing) represent a univariate time series of length $T$ of mean centered VIs at a single location. Note that mean and trend terms can be easily incorporated into the model as separate components, if necessary, so for the purpose of this work we focus solely on modeling seasonality. Minor trend or mean shifts remaining in the data after centering may be captured by the temporal evolution in the seasonality of the DLM. We consider a Bayesian reduced Fourier-form DLM as follows:

$$
\begin{array}{rlrl}
Y_{t} & =\sum_{j=1}^{q} S_{j t}+v_{t} & v_{t} \sim N\left(0, \sigma_{e}^{2}\right) \\
S_{j t} & =\cos \left(\omega_{j}\right) S_{j, t-1}+\sin \left(\omega_{j}\right) S_{j, t-1}^{*}+w_{j t} & \\
S_{j t}^{*} & =-\sin \left(\omega_{j}\right) S_{j, t-1}+\cos \left(\omega_{j}\right) S_{j, t-1}^{*}+w_{j t}^{*} & w_{j t}, w_{j t}^{*} \sim N\left(0, \sigma_{w}^{2}\right) \\
\sigma_{e} & \sim \text { Cauchy }^{+}\left(0, c_{1}\right) & \\
\sigma_{w} & \sim \text { Cauchy }^{+}\left(0, c_{2}\right) \\
\boldsymbol{\theta}_{0} & \sim N_{p}\left(\boldsymbol{m}_{0}, \boldsymbol{C}_{0}\right)
\end{array}
$$

for $t=1, \ldots, T$, and $j=1, \ldots, q$, where $q$ is the number of harmonics and $\boldsymbol{\theta}_{t}=\left(S_{1 t}, S_{1 t}^{*}, \ldots, S_{q t}, S_{q t}^{*}\right)^{T}$ is the $(p=2 q)$-dimensional state vector at time $t$. The observation (2a) and state (2b-2c) equations are well-defined in the DLM literature (see, for example, West and Harrison (1999)). Model (2) is a special case of (1) with time-invariant $V_{t}=V=\sigma_{e}^{2}$, $\boldsymbol{W}_{t}=\boldsymbol{W}=\sigma_{w}^{2} \boldsymbol{I}_{\boldsymbol{p}}, \boldsymbol{F}_{t}=\boldsymbol{F}=\left[\begin{array}{lllll}1 & 0 & \ldots & 1 & 0\end{array}\right]_{1 \times p}$ and $\boldsymbol{G}_{t}=\boldsymbol{G}$ being a $p \times p$ block diagonal matrix, with $j^{\text {th }}$ block

$$
\boldsymbol{G}_{j}=\left[\begin{array}{cc}
\cos \left(\omega_{j}\right) & \sin \left(\omega_{j}\right) \\
-\sin \left(\omega_{j}\right) & \cos \left(\omega_{j}\right)
\end{array}\right] \quad \text { for } j=1, \ldots, q=p / 2, \quad \forall t \in\{1, \ldots, T\} .
$$

$\boldsymbol{G}_{\boldsymbol{j}}$ is a rotation matrix derived so that if $\boldsymbol{W}=\mathbf{0}, S_{1 t}, \ldots, S_{q t}$ are exactly the Fourier harmonics $\left(S_{j t}=a_{j} \cos \left(t \omega_{j}\right)+b_{j} \sin \left(t \omega_{j}\right)\right)$ and $S_{1 t}^{*}, \ldots, S_{q t}^{*}$ are the conjugate harmonics, $\left(S_{j t}^{*}=-a_{j} \sin \left(t \omega_{j}\right)+\right.$ $\left.b_{j} \cos \left(t \omega_{j}\right)\right)$, where $\omega_{j}=2 \pi j / s$ with $s$ being the period.

The variance parameter $\sigma_{w}^{2}$ controls the extent to which the seasonal structure of the model can evolve through time. If $\sigma_{w}^{2}=0$, model (2) reduces to a $q$-harmonic regression that assumes the 
seasonal cycles do not change from year to year. When $\sigma_{w}^{2}>0$ the seasonality is no longer strictly periodic and evolves in time due to the stochastic nature of the evolution equation. Therefore, a $q$-harmonic reduced Fourier-form DLM is more flexible than a $q$-harmonic regression model.

The fully Bayesian specification of the model is completed with independent half-Cauchy priors on $\sigma_{e}$ and $\sigma_{w}$, i.e., Cauchy distributions truncated at zero. The prior specification for the parameters of the variance components of the DLM is particularly important in the context of modeling satellite sensor data when it is desirable to have an automated procedure that requires little to no tuning to implement the methods for a large number of locations. In particular, the degree to which seasonality evolves over time (controlled by $\sigma_{w}$ ) as well as the amount of noise (controlled by $\sigma_{e}$ ) may vary substantially across locations. Therefore, priors on variances (or standard deviations) should aim to be (weakly) uninformative.

We choose the half-Cauchy prior on the standard deviations over the more commonly chosen prior for Bayesian DLMs, the conditionally conjugate inverse-gamma prior on the variances, due to the tendency of the inverse-gamma prior to be informative when a hierarchical variance parameter is small (see, for example, the discussion in Gelman (2006)). If a location exhibits very regular seasonality across years (i.e. no significant changes in phenological patterns), $\sigma_{w}^{2}$ should be very close to 0 , relative to $\sigma_{e}^{2}$, which makes the potential informativity of the inverse-gamma prior undesirable. We illustrate with a small simulation study, provided in Supplementary Materials, that our choice of the half-Cauchy prior is less informative and more robust than the inverse-gamma prior for varying magnitudes of $\sigma_{w}$.

\subsubsection{MCMC Estimation}

The joint posterior distribution $\pi\left(\boldsymbol{\theta}_{0: T}, \boldsymbol{\psi} \mid \boldsymbol{y}_{1: T}\right)$, where $\boldsymbol{\psi}=\left(\sigma_{e}, \sigma_{w}\right)$, of the Bayesian DLM (2) is not analytically tractable, so we use Markov Chain Monte Carlo (MCMC) simulation to approximate this distribution. Specifically, we use a two step Gibbs sampler to alternate draws of $\boldsymbol{\theta}_{0: T}$ and $\psi$. A major motivation for using a two step Gibbs sampler is that, given a draw of $\boldsymbol{\psi}$, a joint draw of $\boldsymbol{\theta}_{0: T}$ can be obtained efficiently from its full conditional distribution $\left(\pi\left(\boldsymbol{\theta}_{0: T} \mid \boldsymbol{\psi}, \boldsymbol{y}_{1: T}\right)=\right.$ $\left.\pi\left(\boldsymbol{\theta}_{T} \mid \boldsymbol{\psi}, \boldsymbol{y}_{1: T}\right) \prod_{t=0}^{T-1} \pi\left(\boldsymbol{\theta}_{t} \mid \boldsymbol{\theta}_{t+1}, \boldsymbol{\psi}, \boldsymbol{y}_{1: T}\right)\right)$ using Forward Filtering Backward Sampling (FFBS) (Carter and Kohn 1994; Frühwirth-Schnatter 1994). The FFBS algorithm can be thought of as a simulation 
version of the smoothing recursions of the Kalman smoother. As a first step, the Kalman filter is run to obtain the distribution, $\pi\left(\boldsymbol{\theta}_{T} \mid \boldsymbol{\psi}, \boldsymbol{y}_{1: T}\right)$ (forward filtering) and a draw of $\boldsymbol{\theta}_{T}$ is obtained from $\pi\left(\boldsymbol{\theta}_{T} \mid \boldsymbol{\psi}, \boldsymbol{y}_{1: T}\right)$. The algorithm then moves backward, for $t=T-1, \ldots, 0$ sequentially sampling $\boldsymbol{\theta}_{t}$ from the conditional distributions, $\pi\left(\boldsymbol{\theta}_{t} \mid \boldsymbol{\theta}_{t+1}, \boldsymbol{\psi}, \boldsymbol{y}_{1: T}\right)$ (backward sampling). The distributions $\pi\left(\boldsymbol{\theta}_{T} \mid \boldsymbol{\psi}, \boldsymbol{y}_{1: T}\right)$ and $\pi\left(\boldsymbol{\theta}_{t} \mid \boldsymbol{\theta}_{t+1}, \boldsymbol{\psi}, \boldsymbol{y}_{1: T}\right), t=T-1, \ldots, 0$, are all Gaussian.

Due to the independent prior specification on $\sigma_{e}$ and $\sigma_{w}$, the joint full conditional posterior distribution of $\boldsymbol{\psi}$ is $\pi\left(\boldsymbol{\psi} \mid \boldsymbol{\theta}_{0: T}, \boldsymbol{y}_{1: T}\right)=\pi\left(\sigma_{e} \mid \boldsymbol{\theta}_{0: T}, \boldsymbol{y}_{1: T}\right) \pi\left(\sigma_{w} \mid \boldsymbol{\theta}_{0: T}, \boldsymbol{y}_{1: T}\right)$. Consequently, we obtain a joint draw of $\boldsymbol{\psi}$ by independently sampling $\sigma_{e}$ and $\sigma_{w}$, conditional on a draw of $\boldsymbol{\theta}_{0: T}$, in the second step of the Gibbs sampler. The full conditional distributions for $\sigma_{e}$ and $\sigma_{w}$ are not analytically tractable, so $\sigma_{e}$ and $\sigma_{w}$ are drawn using two independent Metropolis-Hastings (M-H) steps with inverse-gamma (IG) proposal distributions on the transformed parameters $\eta_{w}=\sigma_{w}^{2}$ and $\eta_{e}=\sigma_{e}^{2}$. Refer to Supplementary Materials for details of the M-H steps, including the specific form of the IG proposal distributions used.

\subsection{Summarizing Phenology}

Smoothed time series of VIs are obtained as the mean structure of the observation equation in the reduced Fourier-form DLM. For the purpose of notation, we define this quantity as $\boldsymbol{S} \boldsymbol{V} \boldsymbol{I}_{t}$, $t=1, \ldots, T$, where $\boldsymbol{S} \boldsymbol{V} \boldsymbol{I}_{t}=\boldsymbol{F} \boldsymbol{\theta}_{t}=\sum_{j=1}^{q} S_{j t}$, for $t=1, \ldots, T$ (i.e. the sum of harmonic terms). $\boldsymbol{S} \boldsymbol{V} \boldsymbol{I}_{t}$ is a linear function of $\boldsymbol{\theta}_{0: T}$, and therefore has a joint posterior distribution, $\pi\left(\boldsymbol{S} \boldsymbol{V I} \boldsymbol{I}_{1: T} \mid \boldsymbol{y}_{1: T}\right)$, that is approximated by applying the function to MCMC simulations of $\boldsymbol{\theta}_{1: T}$. The time-specific medians of a resulting set of $M$ draws of $\boldsymbol{S} \boldsymbol{V} \boldsymbol{I}_{1: T}$ are used to approximate the posterior median of $\boldsymbol{S} \boldsymbol{V} \boldsymbol{I}_{1: T}$ and credible intervals can be constructed using the appropriate empirical quantiles.

Given the approximate posterior distribution of $\boldsymbol{S} \boldsymbol{V I} \boldsymbol{I}_{1: T}$, we proceed to estimate the timing of the phenological events of interest. The fully Bayesian specification and MCMC estimation of the model provides the ideal medium for developing methods which address two major challenges in phenological event identification: 1) determining the appropriate number of growing seasons to summarize when truth is unknown, and 2) providing a measure of uncertainty for the estimated timing of phenological events. The distributions are obtained by identifying timings from individ- 
ual posterior draws of $\boldsymbol{S} \boldsymbol{V} \boldsymbol{I}_{1: T}$, as follows. For a pre-specified rule or set of conditions defining one or more phenological events, the timing of an event of interest is defined as the $t$ corresponding to a $\boldsymbol{S} \boldsymbol{V} \boldsymbol{I}_{t} \in \boldsymbol{S} \boldsymbol{V} \boldsymbol{I}_{1: T}$ that satisfies a pre-specified event identification rule (e.g. maxima/minima, inflection points, etc). Using $M$ posterior draws of $\boldsymbol{S} \boldsymbol{V} \boldsymbol{I}_{1: T}$, we obtain $M$ sets of timings of identified events for each year. The specific form of the identification rule we constructed for the MTCI application is discussed in Section 3.2 .3 , but we emphasize here that the methods presented in the following sections should be applicable to any rule that identifies the timing and magnitude of an event as the time $t$ and value of $\boldsymbol{S} \boldsymbol{V} \boldsymbol{I}_{t}$ that satisfies the rule.

\subsubsection{Identifying the Number of Growing Seasons}

When the number of growing seasons or cropping cycles is unknown, scientifically motivated conditions characterizing a secondary or tertiary growing season are usually incorporated into the identification rule. We provide examples of such conditions within the context of our application in Section 3.2.3. Estimating the timings of events from posterior draws of $\boldsymbol{S} \boldsymbol{V} \boldsymbol{I}_{1: T}$ carries through uncertainty from the posterior distribution. Due to the randomness of the draws, it is possible for one draw to satisfy the conditions for multiple growing seasons in a given year, while another draw may not satisfy the conditions.

We utilize $i=1, \ldots, M$ sets of identified timings of events from $M$ posterior draws of $\boldsymbol{S} \boldsymbol{V} \boldsymbol{I}_{1: T}$ to estimate the probability of identifying $g$ growing seasons in year $d, p_{d}(g)$, under a defined identification rule. Specifically, we estimate $p_{d}(g)$ as the proportion of draws which returned timings of events corresponding to $g$ growing seasons in year $d$. That is,

$$
\hat{p}_{d}(g)=\frac{1}{M} \sum_{i=1}^{M} I\left(\# \text { of seasons identified from } \boldsymbol{S} \boldsymbol{V} \boldsymbol{I}_{1: T}^{(i)} \text { in year } d=g\right)
$$

for $g=0,1, \ldots, G$, where $G$ can be specified as the largest number of growing seasons that can reasonably be expected to occur in the region. A possible decision on the number of growing seasons estimated in year $d$ is to choose $n_{d}=\arg \max \hat{p}_{d}(g)$. The corresponding value, $\hat{p}_{d}\left(n_{d}\right)$, is the estimated conditional probability of determining $n_{d}$ growing seasons in year $d$, given the chosen identification rule. A value of $\hat{p}_{d}\left(n_{d}\right)$ close to one implies that the identification rule consistently suggests a single determination for $n_{d}$, while a low value of $\hat{p}_{d}\left(n_{d}\right)$ implies that it is difficult to 
determine $n_{d}$ under the chosen identification rule.

\subsubsection{Uncertainty Quantification in Timings}

By obtaining $M$ sets of identified timings of events from $M$ posterior simulations of $\boldsymbol{S} \boldsymbol{V} \boldsymbol{I}_{1: T}$, point estimates of event timings and corresponding uncertainty intervals can naturally be constructed as means/medians and as quantile intervals, respectively, from these simulations. However, the specific subset of the $M$ sets of event timings used depends on the determination of the number of growing seasons. Point estimates and uncertainty intervals are obtained as follows:

1. Determine $n_{d}$ using the methods in Section 3.2.1.

2. Subset the collection of $M$ timings to retain only the $M_{d}^{*}$ sets of events that were identified in year $d$ from draws where $n_{d}$ seasons where identified.

3. Point estimates for each event of interest are calculated as the mean or median of $M_{d}^{*}$ timings. Corresponding $(100-\alpha) \%$ quantile intervals are constructed as the floor $(\alpha / 2)$ and $M_{d}^{*}$ floor $(\alpha / 2)$ values of $M_{d}^{*}$ ordered timings.

Note that because for each year, $d$, we only retain the sets of timings obtained from draws where $n_{d}$ growing seasons were identified, $\hat{p}_{d}\left(n_{d}\right)$ is an indicator of the reliability of the estimates and their associated uncertainty intervals, since lower $\hat{p}_{d}\left(n_{d}\right)$ values correspond to lower $M_{d}^{*}$ values (fewer draws used to estimate event timings). Small $\hat{p}_{d}\left(n_{d}\right)$ indicates that the number of growing seasons cannot be conclusively determined under the given identification rule, and likewise there is less certainty in the estimates of the events. We do not claim that the $(100-\alpha) \%$ quantile intervals are true credible intervals, nor do we claim they have $(100-\alpha) \%$ coverage probability. This is because the intervals depend on the form of the chosen identification rule and its parameters $\left(L\right.$ and $k \sigma_{e}$ in our case, defined in 3.2.3 as well as the decision on the number of growing seasons per year, $n_{d}$. They do, however, provide an approximation of the amount of variability in estimates of timings of phenological events. The variability comes from three sources: uncertainty due to noise and due to model uncertainty, and variability due to the form of the identification rule (e.g. an estimate of the timing of the maximum in a curve that plateaus at the peak will have high uncertainty). 


\subsubsection{Phenological Event Identification Rule}

In this work, we focus on the estimation of the timing of two key phenological events. The first, onset of greenness or start of season (SOS), marks the beginning of a growing season when chlorophyll content begins to increase, and the second, peak of greenness (POG), refers to the timing of peak growth during the growing season. Our proposed identification algorithm is an adaptation of the methodology of Dash et al. (2010) and Jeganathan et al. (2010a; 2010b). We define start of season as dominant valleys (local minima) and peak of greenness as dominant peaks (local maxima). Figure 2 provides an idealized example of identifying phenological events under this definition.

The identification rule defines multiple growing seasons by requiring that the set of SOS and POG satisfy two conditions that aim to defend against falsely detecting growing seasons due to minor fluctuations in seasonal structure, or due to random fluctuations in the draws of $\boldsymbol{S} \boldsymbol{V} \boldsymbol{I}_{1: T}$. The first condition implements a minimum growing season duration, $L$, such that the time between two sequential SOS (or two sequential POG) must be at least $L$. The second condition implements a location dependent, minimum allowable difference in VI between neighboring SOS and POG. Specifically, it requires that the difference in VI magnitude between an identified SOS and a POG for a growing season must be larger than $k \sigma_{e}$, where $\sigma_{e}$ is the standard deviation in Equation $2 \mathrm{a}$, and $k$ is a constant that can be tuned to a value that suggests a reasonable number of growing seasons per year. In practice, we used the estimated posterior mode of $\sigma_{e}$ for the value of $\sigma_{e}$ in our identification rule.

The algorithm proceeds by first identifying the set of $\boldsymbol{S} \boldsymbol{V} \boldsymbol{I}_{t}$ that correspond to all local minima and maxima of $\boldsymbol{S} \boldsymbol{V} \boldsymbol{I}_{1: T}$. Then, each identified minimum and maximum is iteratively checked against its temporal neighbors to determine if the difference in magnitude between neighboring minima and maxima is larger than $k \sigma_{e}$ and if the distance between sequentially identified minima and maxima is at least $L$ units apart. The subset of maxima and minima that satisfy all conditions are retained as the set of identified starts of season (minima) and peaks of greenness (maxima) for the location. The timings of the events are the times, $t$, corresponding to the identified $\boldsymbol{S} \boldsymbol{V I} \boldsymbol{I}_{t}$. For the remainder of the paper, the notations SOS and POG will refer specifically to the timing of start 
of season and peak of greenness, respectively.

\section{Application to MTCI Data}

The computational effort required for analyzing remote sensing data is not trivial, since time series can be long and methods should be scalable to large regions. Therefore, all computationally intensive methods, such as MCMC and phenological event detection algorithms, were coded in $\mathrm{C}++$ and implemented in $\mathrm{R}(\mathrm{R}$ Core Team 2015) using the package Rcpp (Eddelbuettel and François 2011).

We implemented the proposed methodology on 1148 locations having natural vegetation as land cover classification (Evergreen, Semievergreen, Moist Deciduous, Dry Deciduous, Coastal Vegetation) as specified using the GLC2000 dataset. While there are a considerable number of agricultural locations (and phenology of agriculture is also of interest), they tend to have much lower signal-to-noise ratios, so for the sake of illustrating the methodology they were not included in the current analysis. We apply the Bayesian reduced Fourier-form DLM (2) separately to each location. In the following, we discuss the selection of the number of Fourier terms, the construction and assessment of convergence of the MCMC sampler, and we summarize the phenology for natural vegetation types over southern India.

\subsection{Selection of Fourier Terms}

The choice of the $q$ harmonics to include in any Fourier based method is an important decision, as the harmonics control the smoothness and form of the model fit. For the types of applications considered in this paper, the ultimate objective is the estimation of phenological events from remote sensing data, for which ground "truth" is not available. This makes the determination of an appropriate $q$ more complex than simply determining the model which provides the best "fit" to the data. For example, a model that may be considered a better fit by criteria that minimize error may be too flexible or "wiggly" to reasonably estimate phenological events.

Jeganathan et al. (2010a; 2010b) applied the discrete Fourier transform to time series of MTCI (separately for each year) over India and found it necessary to retain the first four harmonics to 
reasonably represent seasonality in single growing season locations, while six harmonics were necessary in double or triple season locations. We use these scientifically justified results to motivate our model selection. However, reduced Fourier-form DLMs with non-zero $\sigma_{w}$ are not strictly periodic and can provide a more flexible representation of seasonality with $q$ harmonics than a harmonic regression, particularly in locations exhibiting clear inter-annual variability in seasonality. In many cases, the DLM can provide smoothed representations of the time series with similar flexibility as a harmonic regression model fitted separately by year, but with fewer harmonics.

We considered DLM (2) with $q=1, \ldots, 6$ for a diverse sampling of natural vegetation locations. It was computationally and practically inefficient to attempt to perform model selection for all considered locations, so we selected a sample of locations representing various natural vegetation land cover types, as well as varied seasonal structure. We considered at most $q=6$ harmonics, as we assumed that DLM (2) should not require more harmonics to represent the scientific information than the number found to be necessary by the scientists using the Fourier transform. The statistical importance of individual harmonics $S_{j, 1: T}$ on $\boldsymbol{S} \boldsymbol{V I} \boldsymbol{I}_{1: T}$ for $j=1, \ldots, q$ was assessed by computing the proportion of the $T$ pointwise $99 \%$ credible intervals $\left\{\left(S_{j, t}^{0.5 \%}, S_{j, t}^{99.5 \%}\right), t=1, \ldots, T\right\}$ which did not contain 0 (i.e. $\left.\sum_{t=1}^{T} I\left(0 \notin\left(S_{j, t}^{0.5 \%}, S_{j, t}^{99.5 \%}\right)\right) / T\right)$. We assess overall model adequacy using root mean square error (RMSE) calculated as $R M S E=\sqrt{\sum_{t=1}^{T}\left(y_{t}-S V I_{t}\right)^{2}}$, and the deviance information criterion (DIC). DIC can be thought of as the Bayesian analog of Akaike information criterion (AIC), where the number of parameters in AIC is replaced by an estimate of the effective number of parameters in DIC (Spiegelhalter et al. 2002). Estimates of DIC, RMSE, and the effective number of parameters for models with $q=2, \ldots, 6$ are presented in Table 1 for six example locations ranging in degree of inter-annual variability (characterized by the estimated posterior mode of $\sigma_{w}$ from a model with $q=3$ parameters).

In general, we found that the sensitivity of $\boldsymbol{S} \boldsymbol{V} \boldsymbol{I}_{1: T}$ to the included harmonics in the model depended on the amount of inter-annual variability in seasonality in a location. When seasonality evolved substantially from year to year, model fits were less sensitive to the choice of included harmonics. The majority of considered locations of this type only suggested the use of the first two or three harmonics. Models with two or three harmonics corresponded to low DIC values, 
see for example the values calculated for the bottom three locations included in Table 1, and the proportion of credible intervals which did not contain 0 for $q>3$ harmonics were generally small $(<0.2)$. For these locations, the DLM represents key features in the time series using fewer harmonics than a non-dynamic model and is less prone to small fluctuations that can occur as an artifact from including higher order harmonics in the model. Figure 3 illustrates this by comparing the estimated posterior median $\boldsymbol{S} \boldsymbol{V} \boldsymbol{I}_{1: T}$ from the $q=3$ DLM to the estimated mean from a $q=6$ harmonic regression (the latter fitted separately to each year of data).

For locations which exhibit little change in seasonality across years, the DLM provided a similar fit to a $q$-harmonic regression model and therefore, the choice of included harmonics had higher influence on $\boldsymbol{S} \boldsymbol{V} \boldsymbol{I}_{1: T}$. We found that, in general, the proportion of credible intervals not containing zero was small for harmonics beyond the first three in the $q=6$ DLM for the majority of considered locations of this type, with a few locations suggesting the inclusion of the $4^{\text {th }}$ harmonic. A similar conclusion was drawn comparing DIC across models, where for most considered locations the decrease in DIC from $q=3$ to $q=4$ harmonics was small (see for example the first two locations in Table 1). However, for a small number of considered locations, there was a moderate decrease in DIC from $q=3$ to $q=4$ harmonics as exhibited in the third location included in Table 1. For these locations, we ultimately determined that it was preferable to fit a model with slightly fewer $(q=3)$ harmonics than a larger number, as including additional harmonic terms does not necessarily increase the flexibility of the DLM. In fact, a model with fewer harmonics will often be more flexible than a model including higher order harmonics. This is illustrated by the fact that the effective number of parameters may decrease, and/or RMSE may increase, when more parameters are added to the model. In these cases, the model with a smaller number of harmonics is more flexible since in the DLM a larger evolution variance allows the DLM to accommodate deviations away from a strictly periodic cycle. In Table 1, the decrease in effective number of parameters can be seen in the first three locations from $q=2$ to $q=3$, and the fourth location illustrates that RMSE can increase as harmonics are added to the model.

Figure 4 compares the posterior median of $\boldsymbol{S} \boldsymbol{V} \boldsymbol{I}_{1: T}$ (top) and estimated timings of events and uncertainty intervals (bottom) for models with $q=2, \ldots, 6$ applied to the two locations in Figure 
1. The estimated timings of events, in particular, were fairly robust to the choice of $q$, as demonstrated by the similarity in estimates for $q>2$ in both locations. In summary, using the reduced Fourier-form DLM we found that we were reasonably able to capture seasonality and estimate SOS and POG over the majority of locations using the first $q=3$ harmonics. While we could have considered further tuning the number of harmonics for each location, the decision to fit a model with the same number of harmonics to all locations is motivated by finding a parsimonious model that allows an automatic implementation for a large number of locations.

\subsection{Convergence and Effective Size}

For each natural vegetation grid cell, we estimated the reduced Fourier-form model with $q=3$ harmonics, $s=46, c_{1}=0.1$, and $c_{2}=0.1$, initially running two MCMC chains, in parallel, for 10,000 iterations. Computation time for a complete analysis (from data to phenological event summaries) for one location took $\approx 1.5 \mathrm{~min}$ on a $2.6 \mathrm{GHz}$ Intel Core i5 processor. Convergence of MCMC algorithm was assessed (results not shown) using trace plots and ergodic means for a sample of locations, and by monitoring the Gelman-Rubin diagnostic for all locations since a visual analysis of convergence for over 1,000 locations was unrealistic.

For locations exhibiting clear variability in seasonality, the chains appeared to converge within 1,000 iterations, but in locations with little variability, the chains tended to take longer to converge due to small values causing poor mixing in the chains of $\sigma_{w}$. For these locations, an additional 10,000 iterations were sampled in each chain, to better sample the posterior. The chains were then thinned after burn-in to retain 4,000 samples for estimating the timings of SOS and POG. Inference about summaries of POG and SOS are of primary interest, so autocorrelation in posterior samples of POG and SOS calculated from $M$ draws of $\boldsymbol{S} \boldsymbol{V} \boldsymbol{I}_{1: T}$ was also investigated. The effective sample sizes for POG and SOS were calculated using the coda package in R (Plummer et al. 2006), and found to be nearly equal to $M$, suggesting little to no autocorrelation in samples of POG and SOS.

\subsection{Summary of Findings}

Several values of $L$ and $k$ were considered for the identification rule. We ultimately summarized results using $L=8$ (suggesting that the duration of a growing season must be at least $\approx$ two 
months), and $k=3$ (suggesting that difference in MTCI value between SOS and peak growth must be at least three times more than the estimated standard deviation of the observation error). The values were chosen based on visual assessment for the purpose of illustrating the methodology, but could easily be tuned further given scientific information.

To determine the number of growing seasons per year, we computed the estimates of the probabilities in (3) for $g=0, \ldots, 3$ using 4000 draws from the posterior distribution at each natural vegetation location. The number of POG's identified in one year defines the number of growing seasons, so under our rule the probability in (3) has the specific form

$$
\hat{p}_{d}(g)=\frac{1}{M} \sum_{i=1}^{M} I\left(\# \text { of } P O G^{(i)} \text { in year } d=g\right)
$$

Maps of the estimated probability for each location that two or more growing seasons $\left(\hat{p}_{d}(2)+\right.$ $\left.\hat{p}_{d}(3)\right)$ were identified by the algorithm for years $d=2004-2006$ are shown in Figure 5. Interestingly, the probability of identifying two or more growing seasons appeared to increase between 2004 and 2005 in the western side of the region. This change can be seen in the time series at many locations in this area, suggesting a shift occurred in the phenology between 2004 and 2005. It is unclear whether this change was due to anthropogenic activity or natural occurring events.

Conditional on the number of growing seasons per year, point estimates of POG and SOS were calculated as the median timing of the $i=1, \ldots, M_{d}^{*}$ posterior values of $\mathrm{POG}^{(i)}, \mathrm{SOS}^{(i)}$ for each year, $d$, and uncertainty in the estimates is expressed using $90 \%$ quantile intervals. Figure 6 presents posterior median estimates and $95 \%$ credible intervals for $\boldsymbol{S} \boldsymbol{V} \boldsymbol{I}_{1: T}$, as well as point estimates and corresponding uncertainty intervals of identified SOS and POG for the two locations in Figure 1. Estimates and uncertainty for SOS in terms of days for these two locations are given in Table 2. A composite is represented in dates as 8-day intervals beginning January 1 (e.g. Composite number 1 corresponds to January 1-8). The Evergreen location is an example of a location exhibiting very little inter-annual variability, with consistent estimates of SOS from 2003-2007, while the Semievergreen location shows much more variable estimates and changes in the number of growing seasons across years. Two seasons were identified in 2003 and 2004, while only one growing season is identified in 2005-2007. Uncertainty in POG estimates increase dramatically 
in 2005-2007 as the seasonal structures become plateau-like, making POG difficult to identify precisely.

Averaging across estimates obtained from implementing the methodology on each included location, first SOS in 2005 averaged 5.7 days earlier than in 2004, but in 2006 averaged 9.8 days later than in 2005. These differences are not outside the estimated uncertainty in estimates of SOS and POG, however. The majority of SOS estimates in 2004-2006 had interval widths ranging from 16 to 80 days and the majority of estimated POG had interval widths ranging from 24 to 160 days. Note that since the temporal unit of the data is an 8-day composite, the smallest possible interval width is 8 days. In general, SOS was more precisely estimated than POG. Approximately 80\% of estimated SOS had a $90 \%$ interval width of 48 days or less, while only about $65 \%$ of POG estimates had intervals with widths at least that small.

\section{Conclusions and Future Work}

The approach presented in this paper based on a Bayesian analysis of DLMs provides a natural environment to model and extract phenological patterns from time series of satellite derived VI data, and is a flexible extension of currently applied methods. The methodology is also extendable to other types of satellite sensor data, when the objective is to characterize growth cycles or features of growth cycles. The recursive estimation procedures of Bayesian DLMs using the Kalman Filter and FFBS easily handle missing values, even when data are missing for sequential time points. This eliminates the need to preprocess and impute missing values, and incorporates time dependence to better inform the distribution of states at times when data are missing. Using MCMC simulation of the model, we developed methods to assess the number of growing seasons per year using estimated probabilities and additionally were able to provide uncertainty intervals with estimates of the timings of phenological events, which is novel in this area to our knowledge.

The model utilized in this paper can be extended to allow for more complex modeling. We have assumed a lack of systematic long term trend across years; a constant evolution of both the state and observation equations (by imposing constant $V=\sigma_{e}^{2}$ and constant $\boldsymbol{W}=\sigma_{w}^{2} \boldsymbol{I}$ ); and all harmonics included in the model evolve with the same variance, $\sigma_{w}^{2}$. The first assumption can be 
easily relaxed by adding a either a time-varying or constant mean/trend component to the model. Relaxing the assumption of constant $V$ to time-varying $V_{t}$ may be beneficial since time series of MTCI data seem to exhibit higher variability, or noise, around POG, with inter-annual changes in the magnitude of this variation, particularly in locations with a marked shift in the phenology. This suggests possible dynamic evolution of the observation equation variance. Investigating more flexible specifications of $\boldsymbol{W}_{t}$ is also of interest.

In this work, we have only considered the temporal dependence in the VIs. This approach is massively scalable through embarrassingly parallel computing, making it feasible to create global scale products summarizing the historical evolution of life cycles in vegetated land surfaces. A future direction of this work is to construct a model that incorporates the spatial dependence between locations. While we do not expect that a fully spatio-temporal model will substantially affect the point estimates of the event timings, it may reduce the uncertainty in these estimates. Specific types of models that may be considered include the spatio-temporal dynamic models of Gamerman (2010), Cressie and Wikle (2011), Banerjee et al. (2014), and Gelfand and Banerjee (2017). Several of these rely on basis function approximations for dimension reduction, with subsequent modeling of the basis function coefficients. In phenology, challenges include potentially abrupt land-use differences (with associated phenology differences) both spatially and over time, contributing to substantial space-time non-stationarity. We had static land-use data (a snap-shot in time), however MTCI time series provide evidence of temporal land-use changes in some locations. The latter are reflected in individual locations' temporal trajectories, but possibly not in their spatial neighbors' trajectories. Due to the spatial land-use complexities, and our primary goal being to identify the timing of phenological events (not spatial prediction), we used only the location-specific temporal trajectory of the MTCI data for each location's SOS and POG timing estimates. In the future, dynamic land-use could be incorporated in the model as an explanatory variable in a mixture-type space-time mean structure, and in a non-stationary spatial covariance structure (Schmidt et al. (2011)), although at substantially increased computational cost. We continue to study land-use change estimation in a complementary project. While we concentrated on temporal uncertainty only in this paper, we recognize the importance of spatial structure in un- 
certainty assessment, and the need for continued work to address the complexities of space-time non-stationarity in phenology.

\section{Acknowledgements}

We are grateful to the NERC Earth Observation Data Centre for providing the MTCI data. We thank the Editor, Associate Editor and Referee for valuable comments that led to substantial improvements in the paper. This work was partially supported by the National Science Foundation (DMS-1638521).

\section{References}

Agrawal, S., Joshi, P. K., Shukla, Y., and Roy, P. S. (2003), "SPOT vegetation multi temporal data for classifying vegetation in south central Asia," Current Science, 84(11), 1440-1448.

Atkinson, P. M., Jeganathan, C., Dash, J., and Atzberger, C. (2012), "Inter-comparison of four models for smoothing satellite sensor time-series data to estimate vegetation phenology," Remote Sensing of Environment, 123, 400-417.

Banerjee, S., Carlin, B. P., and Gelfand, A. E. (2014), Hierarchical Modeling and Analysis for Spatial Data, 2 edn, Boca Raton, FL: Chapman and Hall/CRC.

Carter, C. K., and Kohn, R. (1994), “On Gibbs Sampling for State Space Models,” Biometrika, $81(3), 541-553$.

Cressie, N., and Wikle, C. (2011), Statistics for Spatio-temporal Data, Hoboken, N.J: Wiley.

Dash, J., and Curran, P. J. (2004), “MTCI: The MERIS Terrestrial Chlorophyll Index,” International Journal of Remote Sensing, 25(23), 5403-5413.

Dash, J., and Curran, P. J. (2007), "Evaluation of the MERIS terrestrial chlorophyll index (MTCI)," Advances in Space Research, 39(1), 100-104.

Dash, J., Jeganathan, C., and Atkinson, P. M. (2010), "The use of MERIS Terrestrial Chlorophyll 
Index to study spatio-temporal variation in vegetation phenology over India," Remote Sensing of Environment, 114, 1388-1402.

De Beurs, K. M., and Henebry, G. M. (2010), “Spatio-Temporal Statistical Methods for Modelling Land Surface Phenology," in Phenological Research: Methods for Environmental and Climate Change Analysis, eds. M. R. Keatley, and I. L. Hudson, New York: Springer, chapter 9, pp. 177208.

Duncan, J., Dash, J., and Atkinson, P. (2015), “The potential of satellite-observed crop phenology to enhance yield gap assessments in smallholder landscapes," Frontiers in Environmental Science, 3(56).

Eddelbuettel, D., and François, R. (2011), “Rcpp: Seamless R and C++ Integration,” Journal of Statistical Software, 40(8), 1-18.

Frühwirth-Schnatter, S. (1994), "Data Augmentation and Dynamic Linear Models," Journal of Time Series Analysis, 15(2), 183-202.

Gamerman, D. (2010), "Dynamic Spatial Models Including Spatial Time Series," in Handbook of Spatial Statistics, Boca Raton, FL: CRC Press, pp. 437-448.

Geerken, R. A. (2009), “An algorithm to classify and monitor seasonal variations in vegetation phenologies and their inter-annual change," ISPRS Journal of Photogrammetry and Remote Sensing, $64,422-431$.

Geerken, R., Zaitchik, B., and Evans, J. P. (2005), "Classifying rangeland vegetation type and coverage from NDVI time series using Fourier Filtered Cycle Similarity," International Journal of Remote Sensing, 26(24), 5535-5554.

Gelfand, A. E., and Banerjee, S. (2017), "Bayesian Modeling and Analysis of Geostatistical Data," Annual Review of Statistics and Its Application, 4(1), 245-266.

Gelman, A. (2006), "Prior distributions for variance parameters in hierarchical models," Bayesian Analysis, 1(3), 515-533. 
Hanes, J. M., Liang, L., and Morisette, J. T. (2014), "Land Surface Phenology," in Biophysical Applications of Satellite Remote Sensing, ed. J. M. Hanes, Springer Remote Sensing/Photogrammetry, : Springer Berlin Heidelberg, pp. 99-125.

Jeganathan, C., Dash, J., and Atkinson, P. M. (2010a), "Characterising the spatial pattern of phenology for the tropical vegetation of India using multi-temporal MERIS chlorophyll data," Landscape Ecology, 25, 1125-1141.

Jeganathan, C., Dash, J., and Atkinson, P. M. (2010b), "Mapping the phenology of natural vegetation in India using a remote sensing-derived chlorophyll index," International Journal of Remote Sensing, 31(22), 5777-5796.

Jönsson, P., and Eklundh, L. (2004), “TIMESAT - a program for analyzing time-series of satellite sensor data," Computers \& Geosciences, 30, 833-845.

Kalman, R. E. (1960), “A New Approach to Linear Filtering and Prediction Problems," Transactions of the ASME-Journal of Basic Engineering, 82(Series D), 35-45.

Kandasamy, S., and Fernandes, R. (2015), "An approach for evaluating the impact of gaps and measurement errors on satellite land surface phenology algorithms: Application to 20 year NOAA AVHRR data over Canada," Remote Sensing of Environment, 164, 114-129.

O’Connor, B., Dwyer, E., Cawkwell, F., and Eklundh, L. (2012), “Spatio-temporal patterns in vegetation start of season across the island of Ireland using the MERIS Global Vegetation Index," ISPRS Journal of Photogrammetry and Remote Sensing, 68, 79-94.

Petris, G., Petrone, S., and Campagnoli, P. (2009), Dynamic Linear Models with R, Use R!, : Springer New York.

Plummer, M., Best, N., Cowles, K., and Vines, K. (2006), “CODA: Convergence Diagnosis and Output Analysis for MCMC," R News, 6(1), 7-11.

Prasad, S. N., and Hegde, M. (1986), "Phenology and seasonality in the tropical deciduous forest of Bandipur, South India,” Proceedings: Plant Sciences, 96(2), 121-133. 
R Core Team (2015), R: A Language and Environment for Statistical Computing, R Foundation for Statistical Computing, Vienna, Austria.

Sakamoto, T., Yokozawa, M., Toritani, H., Shibayama, M., Ishitsuka, N., and Ohno, H. (2005), “A crop phenology detection method using time-series MODIS data," Remote Sensing of Environment, 96(3-4), 366-374.

Schmidt, A. M., Guttorp, P., and O'Hagan, A. (2011), “Considering covariates in the covariance structure of spatial processes," Environmetrics, 22(4), 487-500.

Spiegelhalter, D. J., Best, N. G., Carlin, B. P., and Van Der Linde, A. (2002), "Bayesian measures of model complexity and fit," Journal of the Royal Statistical Society: Series B (Statistical Methodology), 64(4), 583-639.

Tan, B., Morisette, J. T., Wolfe, R. E., Gao, F., Ederer, G. A., Nightingale, J., and Pedelty, J. A. (2011), “An Enhanced TIMESAT Algorithm for Estimating Vegetation Phenology Metrics From MODIS Data," IEEE Journal of Selected Topics in Applied Earth Observations and Remote Sensing, 4(2), 361-371.

Vrieling, A., de Beurs, K. M., and Brown, M. E. (2011), "Variability of African farming systems from phenological analysis of NDVI time series," Climatic Change, 109(3), 455-477.

Vrieling, A., De Leeuw, J., and Said, M. Y. (2013), "Length of growing period over Africa: Variability and trends from 30 years of NDVI time series," Remote Sensing, 5(2), 982-1000.

West, M., and Harrison, J. (1999), Bayesian Forecasting and Dynamic Models, Springer Series in Statistics, : Springer New York.

Zhang, X., Friedl, M. A., Schaaf, C. B., Strahler, A. H., Hodges, J. C., Gao, F., Reed, B. C., and Huete, A. (2003), "Monitoring vegetation phenology using MODIS," Remote Sensing of Environment, 84, 471-475. 

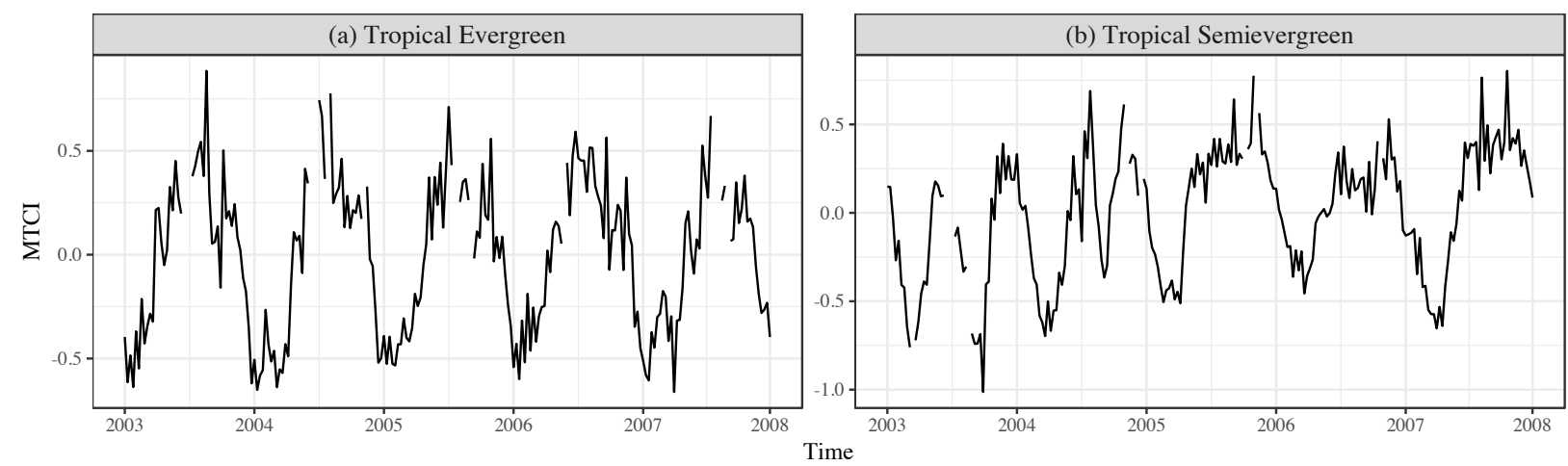

Figure 1: Time series of MTCI for two sample pixels. (a) is an example of a location exhibiting little change in seasonality, while (b) shows inter-annual variability in seasonality over time.

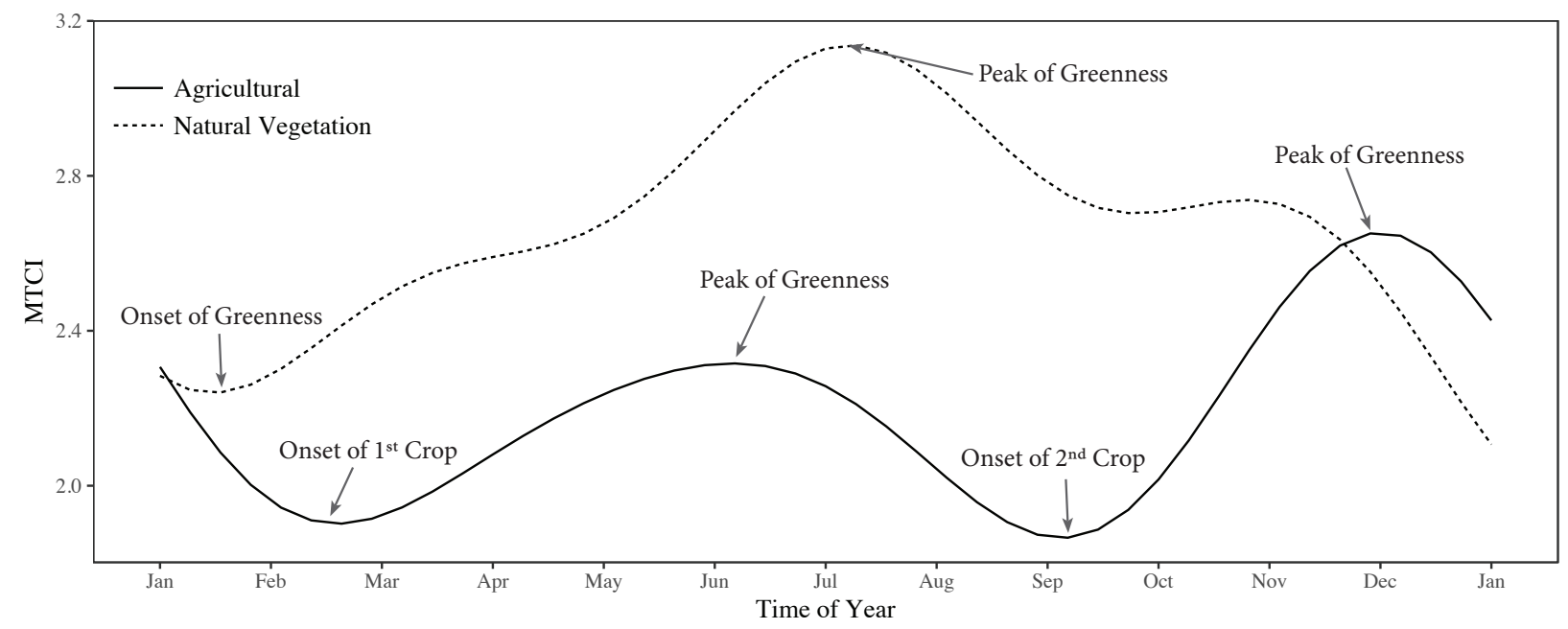

Figure 2: Example representation of idealistic identification of onsets and peaks of greenness from smoothed time series of satellite sensor data. The algorithm should be able to distinguish between a secondary growing season (exhibited in the agricultural series) and a residual peak due to fluctuations in the time series (like the secondary peak in the natural vegetation series.) 


\begin{tabular}{c|r|rrrrr}
\hline \hline Location & Metric & $q=2$ & $q=3$ & $q=4$ & $q=5$ & $q=6$ \\
\hline \hline Moist & DIC & -147.86 & -165.23 & -167.31 & -167.22 & -162.78 \\
Deciduous & RMSE & 2.79 & 2.93 & 2.96 & 2.97 & 2.96 \\
$\hat{\sigma}_{w}=0.0025$ & Eff. Params & 49.06 & 19.30 & 24.45 & 23.92 & 27.50 \\
\hline \hline Coastal & DIC & -136.53 & -152.06 & -153.31 & -153.23 & -151.89 \\
Vegetation & RMSE & 3.21 & 3.20 & 3.17 & 3.22 & 3.20 \\
$\hat{\sigma}_{w}=0.0027$ & Eff. Params & 31.66 & 23.02 & 21.56 & 24.46 & 27.10 \\
\hline \hline Tropical & DIC & -157.34 & -176.95 & -193.35 & -194.89 & -191.81 \\
Evergreen & RMSE & 5.95 & 5.91 & 5.88 & 5.88 & 5.89 \\
$\hat{\sigma}_{w}=0.004$ & Eff. Params & 16.69 & 12.88 & 17.30 & 24.08 & 23.67 \\
\hline \hline Moist & DIC & -72.76 & -65.23 & -45.08 & -21.53 & 17.36 \\
Deciduous & RMSE & 2.74 & 2.74 & 2.77 & 2.75 & 2.75 \\
$\hat{\sigma}_{w}=0.032$ & Eff. Params & 46.73 & 50.63 & 50.88 & 50.45 & 33.40 \\
\hline \hline Tropical & DIC & -217.46 & -214.63 & -213.87 & -205.48 & -194.74 \\
Semievergreen & RMSE & 4.06 & 4.02 & 4.09 & 4.11 & 4.10 \\
$\hat{\sigma}_{w}=0.032$ & Eff. Params & 60.60 & 61.74 & 65.44 & 70.90 & 75.06 \\
\hline \hline Tropical & DIC & -270.12 & -270.65 & -267.47 & -259.85 & -249.62 \\
Semievergreen & RMSE & 1.39 & 1.34 & 1.34 & 1.32 & 1.31 \\
$\hat{\sigma}_{w}=0.036$ & Eff. Params & 68.23 & 74.59 & 76.11 & 80.52 & 86.86 \\
\hline \hline
\end{tabular}

Table 1: Deviance Information Criterion (DIC), root mean squared error (RMSE) and effective number of parameters (Eff. Param) calculated for models with $q=2, \ldots, 6$ included harmonics for six example locations. Three locations (top) correspond to time series where there is very little inter-annual variability, quantified by estimated $\sigma_{w}$ from the model with $q=3$ parameters. The other three locations (bottom) have relatively large estimated $\sigma_{w}$ from the $q=3$ model, representing time series with substantial temporal variability. The two locations highlighted in bold are the two locations used throughout the manuscript to illustrate the methodology. 


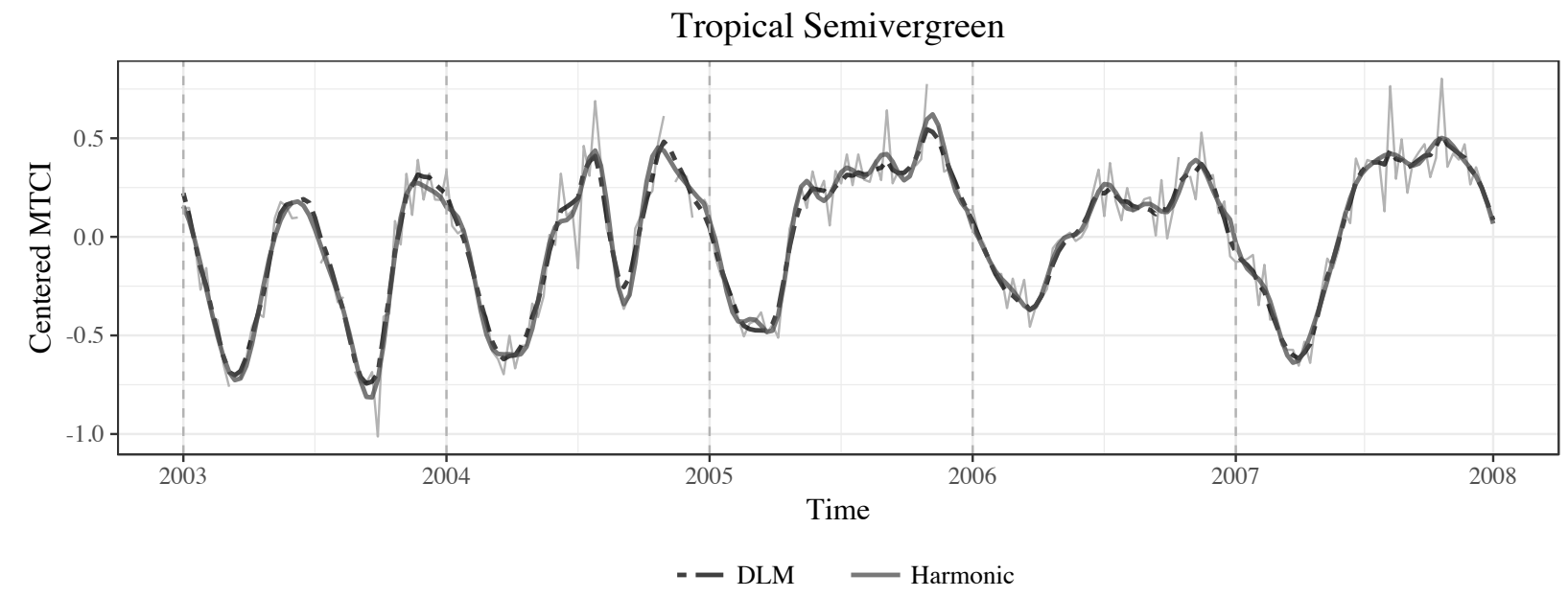

Figure 3: Smoothed curves are compared from a $q=3$ reduced Fourier-form DLM (dashed) and a $q=6$ harmonic regression fit separately to each year (solid). The curves have similar forms, but the non-dynamic model exhibits undesirable artifacts from the higher order harmonics, (e.g. year 2005). 


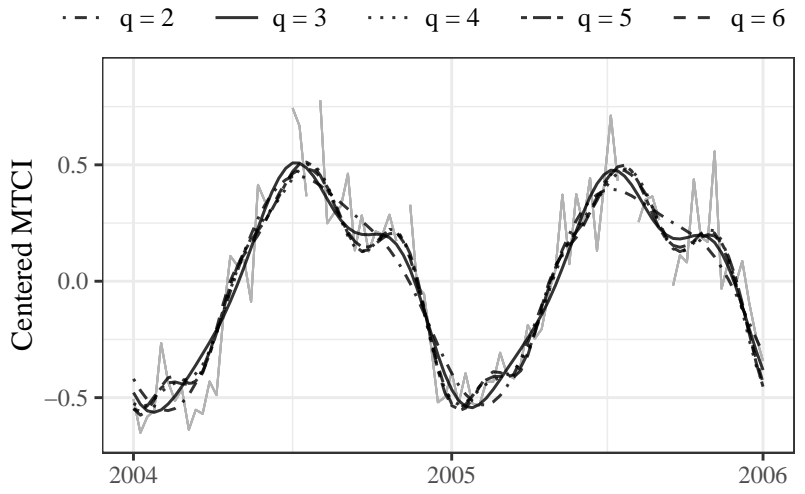

(a)

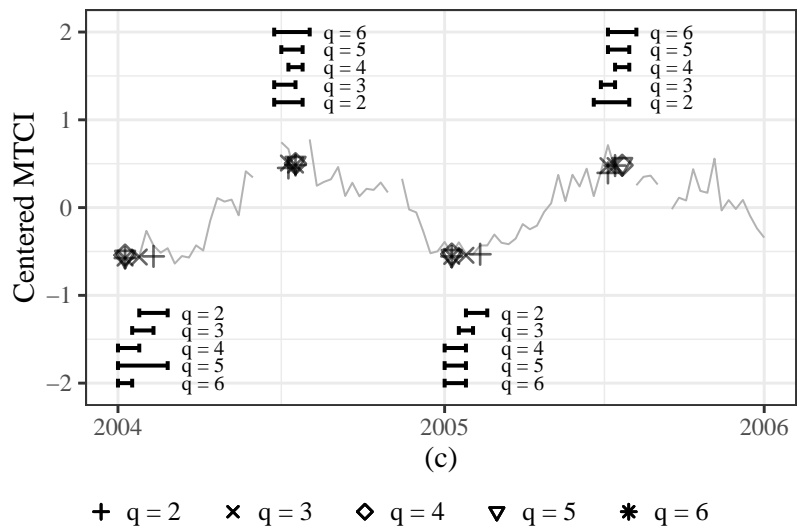

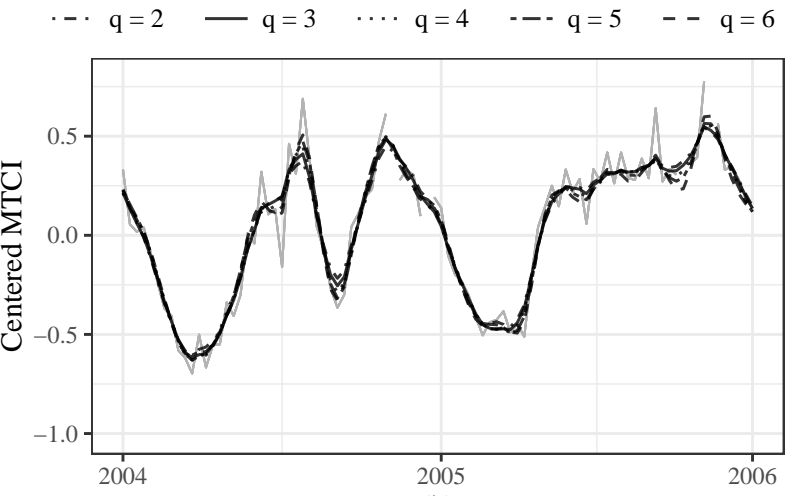

(b)

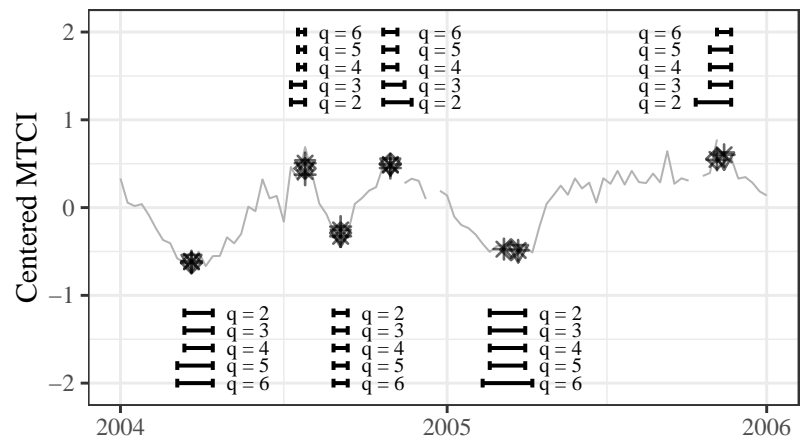

(d)

$$
+\mathrm{q}=2 \times \mathrm{q}=3 \diamond \mathrm{q}=4 \quad \boldsymbol{\nabla} \mathrm{q}=5 \quad * \mathrm{q}=6
$$

Figure 4: Plots (a)-(b) show posterior median estimates of $\boldsymbol{S} \boldsymbol{V} \boldsymbol{I}_{1: T}$ for DLMs with $q=2, \ldots, 6$ for the two locations in Figure 1, respectively. Plots (c)-(d) show the estimates of POG and SOS with uncertainty interval widths shown as horizontal bars, for each of the six models. Only two years (2004 - 2005) are shown for clarity. 

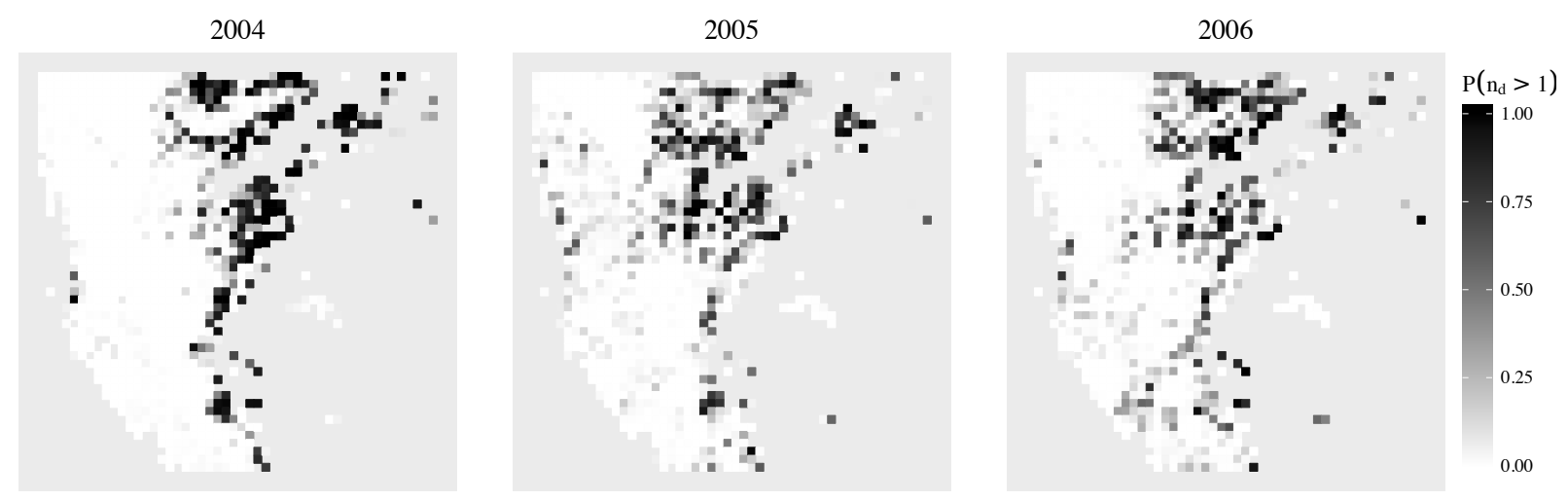

Figure 5: Maps of the probability of identifying more than one growing season for years 2004 2006, estimated for each considered natural vegetation location. 
(b) Tropical Evergreen

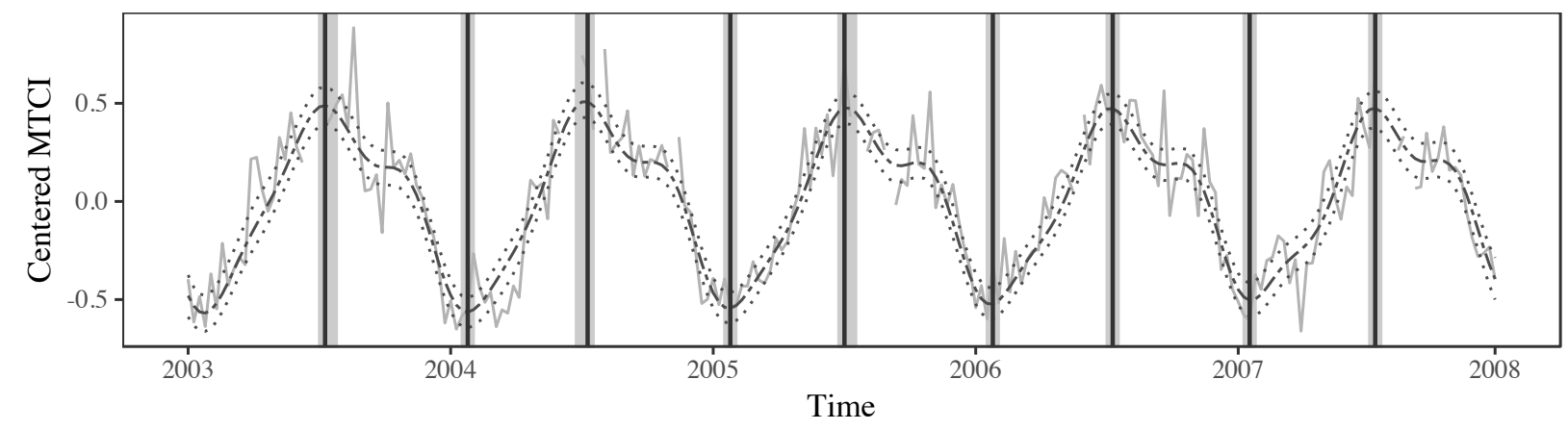

(b) Tropical Semievergreen

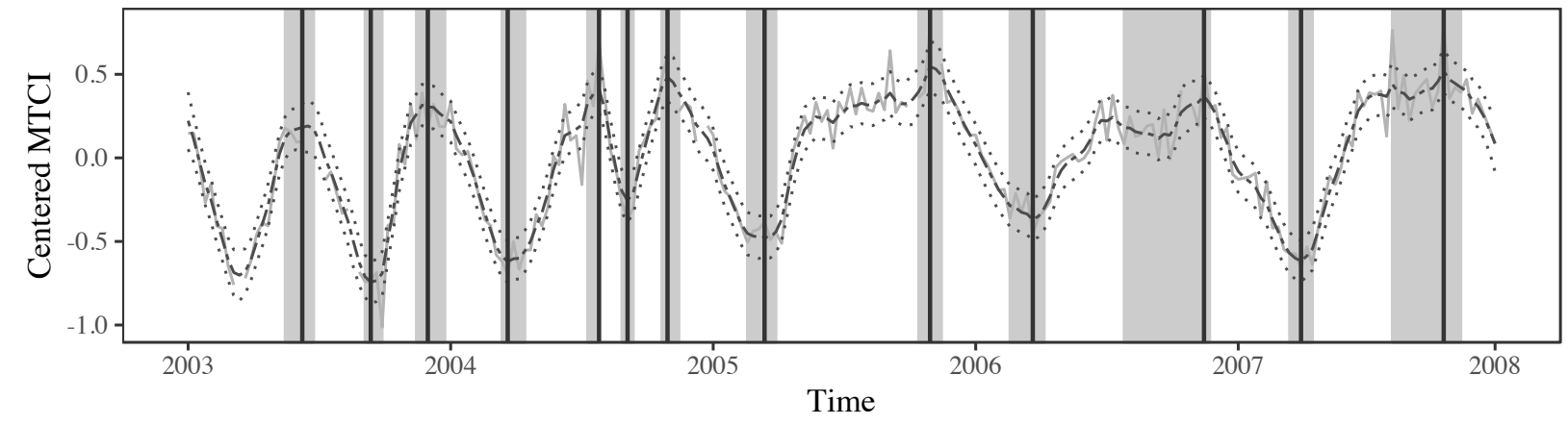

Figure 6: Example of phenological variable estimation for the two sample locations in Figure 1 Posterior median estimates of $\boldsymbol{S} \boldsymbol{V} \boldsymbol{I}_{1: T}$ (dashed) with $95 \%$ credible intervals (dotted) are plotted. The vertical black lines represent the posterior median estimates of POG and SOS, with the width of their respective $90 \%$ intervals visualized with vertical rectangles (grey). Plot (a) illustrates a location with one growing season identified per year, while plot (b) shows a location with substantial inter-annual seasonal variability. 
(a) Evergreen

(b) Semievergreen

\begin{tabular}{c|cc|cc}
\hline Year & Estimate & Interval & Estimate & Interval \\
\hline 2003 & Jul 12 - 19 & Jul 04 - Aug 04 & Jun 10 - 17 & May 17 - Jul 03 \\
& & & Dec 03 - 10 & Nov 17 - Jan 03 \\
\hline 2004 & Jul 11 - 18 & Jun 25 - Jul 26 & Jul 27 - Aug 03 & Jul 11 - Aug 03 \\
& & & Oct 31 - Nov 07 & Oct 23 - Nov 23 \\
\hline 2005 & Jul 04 - 11 & Jun 26 - Jul 27 & Nov 01 - 08 & Oct 16 - Nov 24 \\
\hline 2006 & Jul 12 - 19 & Jun 04 - Jul 27 & Nov 17 - 24 & Jul 28 - Dec 02 \\
\hline 2007 & Jul 12 - 19 & Jun 04 - Jul 27 & Oct 16 - 23 & Aug 05 - Nov 16 \\
\hline
\end{tabular}

Table 2: Posterior estimates, 90\% intervals and interval widths for POG in days for years 2003 - 2007 for the two locations in Figure 6. Posterior estimates are listed as the 8-days corresponding to estimated composite number, and intervals are converted to days by listing the first day corresponding to the lower bound composite and the last day of the upper bound composite. 